\title{
The BGS magnetic field candidate models for the 10th generation IGRF
}

\author{
Vincent Lesur, Susan Macmillan, and Alan Thomson \\ British Geological Survey, Murchison House, West Mains Road, Edinburgh, EH9 3LA, U.K.
}

(Received January 7, 2005; Revised April 27, 2005; Accepted May 27, 2005)

\begin{abstract}
In this paper we describe the derivation of the BGS candidate models for the 10th generation International Geomagnetic Reference Field. Our data set comprised quiet night-time data from the Ørsted and Champ satellites spanning 1999.2-2004.6 and observatory hourly means spanning 1999.0-2004.0. To improve the secular variation estimates for 2005.0-2010.0, predictions based on application of linear prediction filters to long series of observatory annual means were also used. These data were fitted by a spherical harmonic "parent" model with an internal field of maximum degree 36, a quadratic dependence on time up to degree 8 , a linear dependence on time up to degree 12, an external field of maximum degree 2 with linear dependence on time, annual and semi-annual variations, and $D s t$ dependence for degree 1 terms. Additionally for the external field, non-zonal degree 1 coefficients in the Geocentric Equatorial Inertial reference frame with annual variations and dependence on the Interplanetary Magnetic Field $Y$-component are included. The candidate models were then based, for the main field, on an extrapolation to 2005.0 of the truncated parent model, and for the secular variation, on its extrapolation to 2007.5. This latter set of coefficients was then used to generate a synthetic data set at the Earth's surface and this set was augmented with long term linear predictions of observatory annual means, to produce the final candidate secular variation model at 2007.5.
\end{abstract}

Key words: IGRF, geomagnetic field, geomagnetic secular variation.

\section{Introduction}

The International Geomagnetic Reference Field (IGRF) is a model of the Earth's magnetic field widely used in academic and commercial applications. This reference field model is determined by a group of geomagnetic field modellers associated with the International Association of Geomagnetism and Aeronomy (IAGA) Division V, and consists of series of field models defined for given epochs plus models of the magnetic field variation with time: the secular variation $(s v)$. A magnetic field model for epoch 2005.0 and a secular variation model valid for 2005.0 to 2010.0 are required for the 10th generation IGRF.

Never before has such a large quantity of high-quality vector satellite data, spanning almost five years, been available to build magnetic models. However, projecting models forward in time can be unstable, and relatively minor differences in modelling techniques may lead to significant differences in the resulting models. The British Geological Survey (BGS) approach in building candidate models for the IGRF was to use as much available data as possible, and, in particular, hourly mean as well as annual mean observatory data. The effect of introducing observatory data was carefully checked by comparing, at each stage of the modelling process, the BGS candidates with similar models built without observatory data.

The BGS candidates for the IGRF-10 were built in two stages. In the first stage a magnetic field model (the "parent" model) was constructed from satellite and observatory data selected between 1999.0 and 2004.58. This model was

(c) NERC, 2005. All Rights Reserved. then extrapolated forward in time to derive a main field model at 2005.0 and a secular variation model at 2007.5. Truncating this main field model provided the BGS main field candidate for IGRF-10. The secular variation component of the model was used only to synthesize data on a grid at the Earth's surface and this synthetic data set was combined with secular variation data linearly predicted using long time series data from observatories. The BGS secular variation model candidate for 2005.0 to 2010.0 was then estimated by a least squares fit to these two data sets.

The following sections describe the data selection, model parameterisation, data weighting and model estimation. The final section describes how the IGRF candidate models were extracted from the parent model.

\section{Data Selection}

\subsection{Satellite data}

All available Ørsted and Champ scalar and vector data were used. Data were selected $20 \mathrm{~s}$ apart, when $K p \leq 1+$, for Interplanetary Magnetic Field (IMF) components $|B x|$, $|B y| \leq 10 \mathrm{nT}$ and $0 \leq B z \leq 6 \mathrm{nT}$, a maximum solar wind speed of $450 \mathrm{~km} / \mathrm{s}$ and, up to July 2004, Dst index values in the range $-20 \mathrm{nT}$ to $0 \mathrm{nT}$. No real-time $D s t$ indices were used at any point in the model as these are produced by an entirely automatic procedure, sometimes not using the full complement of observatories and therefore contain spikes and baseline shifts. Data with $K p>1+$ in the previous three hour interval before the acquisition time or data with $0<D s t$ or $D s t<-20 \mathrm{nT}$ in the hour before the acquisition time were also not used. Contamination by the magnetic field generated in the ionosphere was minimised at low and mid-latitude by choosing only night-side data 
between 23:00 and 05:00 local time. Only vector data were selected in the geomagnetic latitude range $-55^{\circ}$ to $55^{\circ}$. Otherwise, at higher latitudes only scalar data were selected using the further constraint of maximum polar cap (PC) index $\leq 0.2$ (with this limit chosen by examining the $r m s$ fit to the data as a function of maximum PC). These two latter selection criteria were set to minimise the effect of currents flowing in and above the ionosphere over the polar area. After 2003.0 the maximum solar wind speed limit was allowed to increase to $550 \mathrm{~km} / \mathrm{s}$, as a result of the higher wind speed average for that year, in order to provide enough data.

Ørsted and Champ data up to the end of September 2004 and the end of June 2004 respectively were downloaded but the local-time selection criterion limited the span of Ørsted data at 2004.58. The span of Champ data was also limited, at 2004.3, by the attitude corrections available (Maus, personal communication, McLean et al., 2004). We note that the distributions in time of the Ørsted satellite data, and to a lesser extent the Champ data, have gaps in them due to the local-time selection criterion.

\subsection{Observatory hourly mean data}

Hourly mean vector data at 151 geomagnetic observatories were selected. Any measured discontinuities in these data were applied. Any other discontinuities were dealt with by splitting the observatory series to allow separate biases to be solved for in the model. The selection criteria were similar to those for satellite data: $K p \leq 1+$, IMF component $B z \geq 0$, Dst index values in the range $-15 \mathrm{nT}$ to $0 \mathrm{nT}$ and local time between 23:00 and 05:00. The distribution in time of observatory data starts at 1999.0, stops at 2004.0 and is more even than for satellite data.

In an attempt to minimise the noise level due to currents flowing in and above the polar ionosphere, vector data from observatories at geomagnetic latitudes outside the range $\left[-55^{\circ} ; 55^{\circ}\right]$ were projected onto the direction of an existing magnetic field model based on satellite data only. The equivalent usual practice for satellite data is to use only total intensity values at high latitudes as they are less affected by field aligned current systems than vector data values. However this is not possible for observatory data because an offset has to be introduced for each observatory component to account for the local, un-modelled, crustal field. By using projected data, a linear relationship is maintained between data and observatory offsets.

\subsection{Observatory secular variation data}

Prediction of secular variation to 2010.0 was made using linear prediction filters applied to 159 series of first differences of observatory annual means in $X, Y$ and $Z$ (Macmillan and Quinn, 2000). The time intervals covered by observatory time series are long compared to the time interval of data used in the parent model, with some data extending back into the 19th century. Linear prediction is successful at extrapolating signals that are smooth and oscillatory, though not necessarily periodic, and tests have shown that when predicting more than about 3 years ahead, this method is better than linear regression applied to recent first differences. The time series from 159 observatories were prepared using the file of annual means maintained by BGS, taking account of any jumps and gaps in the data, and discounting certain early parts of records where the noise levels are particularly high. The data used in the final secular-variation model were averages of the predictions for 2005.0-2010.0 and were assigned uncertainties that reflected the past success of prediction for the data series in question. Data from 29 observatories which had time series too short (i.e. less than 10 years) for the application of the linear prediction filter were still used, by computing average secular-variation estimates and assuming that these did not change with time.

\section{Model Parameterisation}

Away from its sources, the magnetic field $\boldsymbol{B}$ is a potential field and therefore can be written as the negative gradient of a potential $\boldsymbol{B}(\theta, \varphi, r, t)=-\nabla V(\theta, \varphi, r, t)$. This potential can be expanded in terms of spherical harmonics:

$$
\begin{aligned}
& V(\theta, \varphi, r, t) \\
& =a\left\{\sum _ { l = 1 } ^ { l i } \sum _ { m = 0 } ^ { l } \left(g_{l}^{m}(t) \cos (m \varphi)\right.\right. \\
& \left.\left.+h_{l}^{m}(t) \sin (m \varphi)\right)\left(\frac{a}{r}\right)^{l+1} \mathrm{P}_{l}^{m}(\cos \theta)\right\} \\
& +a\left\{\sum _ { l = 1 } ^ { l e } \sum _ { m = 0 } ^ { l } \left(q_{l}^{m}(t) \cos (m \varphi)\right.\right. \\
& \left.\left.\quad+s_{l}^{m}(t) \sin (m \varphi)\right)\left(\frac{r}{a}\right)^{l} \mathrm{P}_{l}^{m}(\cos \theta)\right\}
\end{aligned}
$$

where $a(6371.2 \mathrm{~km})$ is the Earth's reference radius, $(\theta, \varphi, r)$ are spherical coordinates in a geocentric reference frame, $P_{l}^{m}(\cos \theta)$ are the Schmidt semi-normalized Legendre functions, and $\left(g_{l}^{m}(t), h_{l}^{m}(t)\right)$ and $\left(q_{l}^{m}(t), s_{l}^{m}(t)\right)$ are the time-dependent Gauss coefficients describing internal and external sources respectively. We have used $l i=36$ and $l e=2$ as the truncation level of the internal and external fields respectively. This maximum internal degree is high and we believe that our estimates of the Gauss coefficients are robust at least up to degree 20 .

The internal Gauss coefficients from degree 1 to 8 are assumed to have a quadratic dependence on time:

$$
\begin{aligned}
& g_{l}^{m}(t)=g_{l}^{m}+\dot{g}_{l}^{m}\left(t-t_{0}\right)+\ddot{g}_{l}^{m}\left(t-t_{0}\right)^{2} \\
& h_{l}^{m}(t)=h_{l}^{m}+\ddot{h}_{l}^{m}\left(t-t_{0}\right)+\ddot{h}_{l}^{m}\left(t-t_{0}\right)^{2}
\end{aligned}
$$

where the time is given in decimal year and $t_{0}$ is the reference date of the model (2002.0). From degree 9 up to 12 a linear dependence on time of the internal Gauss coefficients is assumed, and, for higher degrees, the internal Gauss coefficients are taken as constant with time. The external Gauss coefficients dependence on time is set as linear.

Other authors (Sabaka et al., 2002; Olsen, 2002) have shown that both external and internal degree 1 and 2 Gauss coefficients have a seasonal variation. The same seasonal parameterisation is used here. Let $\tilde{g}_{l}^{m}(t)$ be the parts of the $g_{l}^{m}(t)$ coefficients that account for these seasonal variations, then:

$$
\begin{aligned}
\tilde{g}_{l}^{m}(t)= & \tilde{g}_{1,1 c}^{m} \cos \left(2 \pi\left(t-t_{0}\right)\right)+\tilde{g}_{1,1 s}^{m} \sin \left(2 \pi\left(t-t_{0}\right)\right) \\
& +\tilde{g}_{1,2 c}^{m} \cos \left(4 \pi\left(t-t_{0}\right)\right) \\
& +\tilde{g}_{1,2 s}^{m} \sin \left(4 \pi\left(t-t_{0}\right)\right)
\end{aligned}
$$


where underscripts " $s$ " and " $c$ " denote sine and cosine respectively. Similar representations are used for $h_{1}^{m}(t)$, $q_{1}^{m}(t), s_{1}^{m}(t)$ and for the degree 2 internal and external Gauss coefficients.

A Dst dependence for the degree 1 Gauss coefficients is introduced to represent the variability of the magnetospheric ring current. We used the internal/external Dst separation due to Maus and Weidelt (2004) up to 30 June 2004. Otherwise, a fixed ratio of 0.27 (Langel and Estes, 1985) between the external Dst dependence and the associated internal induced contribution was assumed.

The data set contains observatory data and we have to introduce offsets at each observatory to take into account the field, mainly generated in the crust, which cannot be described by our model. At an observatory, the magnetic field $\boldsymbol{B}$ is:

$$
\boldsymbol{B}(\theta, \varphi, r, t)=-\nabla V(\theta, \varphi, r, t)+\boldsymbol{O}(\theta, \varphi, r)
$$

where the offset vector $\boldsymbol{O}(\theta, \varphi, r)$ is constant in time. Therefore, there are three new parameters per observatory in the geomagnetic latitude range $-55^{\circ}$ to $55^{\circ}$. Outside this range, the data we use are projections of the measured magnetic field onto a priori directions. This a priori model was built for epoch 2002.0 from satellite data only. In these cases, only one parameter is introduced per observatory to account for the crustal offset into this same a priori direction.

The above parameterization of the magnetic field is made in the geocentric reference frame (GEO) that is rotating with a $24 \mathrm{~h}$ periodicity around its $Z$-axis (i.e. the Earth's rotation axis) relative to the Sun-Earth direction. Therefore, any large-scale external field directly linked to the Sun-Earth system will have an apparent $24 \mathrm{~h}$ periodicity in its components perpendicular to the Earth's rotation axis. These components are not included in the above model and directly map into the residuals. In the special case where only night-side data are used these un-modelled components are seen as non-potential fields. One could expect large-scale external field time variations to be fully captured by the $D s t$ index. But the removal of the $S q$ contributions and the averaging process over the four observatories, which are part of the Dst derivation process, minimizes these components. On the other hand, the large-scale external field component along the Earth's rotation axis does not have this apparent $24 \mathrm{~h}$ periodicity and can fully contribute to the Dst index. (In this short description we neglect the angle between the magnetic dipole axis and the Earth's rotation axis.) The fact that the four observatory contributions to the Dst index are "normalized to the dipole equator" makes the problem even more complex. Lesur et al. (2005) presented evidence of large-scale external magnetic fields that do not contribute to the Dst index but nevertheless produce a detectable signal in the near-Earth environment. This magnetic signal correlates well in time with the $Y$ component of the IMF (IMF $B_{y}$ ) in the Geocentric Solar Magnetospheric (GSM) system of coordinates. We modelled the contributions of these fields in the Geocentric Equatorial Inertial (GEI) reference frame. This system is (to the first order) fixed with respect to the distant stars, its $Z$ axis lies along the Earth's rotation axis and its $X$-axis points towards the first point of Aries (Hapgood, 1992). In the GEI system the Sun-Earth vector rotates with only a yearly periodicity. In this system of coordinates we therefore define a potential field for the components perpendicular to the Earth's rotation axis:

$$
\begin{aligned}
& V^{\text {gei }}(\theta, \varphi, r, t) \\
& \quad=r\left(q_{1}^{1 \mathrm{gei}}(t) \cos (\varphi)+s_{1}^{1 \mathrm{gei}}(t) \sin (\varphi)\right) \mathrm{P}_{1}^{1}(\cos \theta)
\end{aligned}
$$

where $(\theta, \varphi, r)$ are the spherical coordinates in GEI that differ from the coordinates in GEO only by the $\varphi$ values. Finally, we define the time behaviour of $q_{1}^{1 g e i}(t)$ as:

$$
\begin{aligned}
q_{1}^{1 g e i}(t)= & \left(q_{1,1 c}^{1 c}+q_{1,1 c}^{1 i} B_{y}\right) \cos \left(2 \pi\left(t-t_{0}\right)\right) \\
& +\left(q_{1,1 s}^{1 c}+q_{1,1 s}^{1 i} B_{y}\right) \sin \left(2 \pi\left(t-t_{0}\right)\right)
\end{aligned}
$$

and similarly for $s_{1}^{1 g e i}(t)$. In equation $6, B_{y}$ is the IMF $Y$ component in GSM. This parameterisation in GEI coordinates is not entirely satisfactory because the (very small amplitude) $24 \mathrm{~h}$ periodicity in the magnetic field component along the Earth's rotation axis, which is due to the rotation of the magnetic pole, is not modelled. An alternative approach that uses GSM coordinates is not totally satisfactory either because of the complex behaviour of this system relative to GEO. Introducing this parameterisation in GEI is important for resolving variations in the large-scale external fields at time intervals less than $24 \mathrm{~h}$. We tried, but did not succeed in extracting from the data set the associated induced field: this cannot be readily separated from the magnetic field generated in the core.

\section{Data Weighting, Attitude Errors and Covari- ance Matrix}

The standard deviations (SDs) associated with both satellite and observatory data were defined as:

$$
\sigma=\sigma_{0}+d_{z}(1+\cos (z a))
$$

where $z a$ is the zenith angle of the Sun (i.e. the angle between the local vertical and the direction of the sun), and the factor $d_{z}$ is set to $3 \mathrm{nT}$ for satellite scalar data and to $20 \mathrm{nT}$ for observatory projected data. Otherwise $d_{z}$ is set to $0 \mathrm{nT}$ and $\sigma_{0}$ to $2 \mathrm{nT}$ for all vector data. The dependence relative to the zenith angle was introduced to account for the noise level due to the increased conductivity of the sun-lit ionosphere.

As in Holme (2000) and Olsen (2002), we account for the anisotropy in the attitude accuracy of Ørsted vector data by estimating off-diagonal elements of the data covariance matrix.

No further selection or decimation of the data was introduced to deal with the high data density at high latitudes. Instead, the data values were multiplied by weights. The spherical surface was divided into roughly equal-area cells whose size at the equator was $5^{\circ}$ in latitude and longitude. For a data point in a given cell, a weight was calculated as the ratio of the average number of data in non-empty cells to the number of data in that cell. These weights were computed independently for observatory and satellite data. To introduce these weights into our parameter estimation 
Table 1. Mean and rms misfit to the data set for the parent model (nT) and the model estimated without the observatory data.

\begin{tabular}{lcccccccc}
\hline & \multicolumn{3}{c}{ Parent model } & & \multicolumn{3}{c}{ Fit without observatory data } \\
\cline { 2 - 4 } \cline { 6 - 8 } Component & $N$ & mean & rms & & $N$ & mean & rms \\
\hline$X$ satellite & 60,931 & 0.49 & 6.45 & & 60,931 & 0.28 & 6.33 \\
$Y$ satellite & 60,931 & 0.27 & 5.80 & & 60,931 & 0.20 & 5.68 \\
$Z$ satellite & 60,931 & -0.27 & 5.00 & & 60,931 & 0.24 & 4.84 \\
$F$ Satellite & 14,004 & -2.25 & 6.75 & & 14,004 & -0.97 & 6.62 \\
$X$ observatory & 75,652 & 0.00 & 5.39 & & - & - & - \\
$Y$ observatory & 75,652 & 0.00 & 4.65 & & - & - & - \\
$Z$ observatory & 75,652 & 0.00 & 3.54 & & - & - & - \\
$F$ observatory & 35,653 & 0.57 & 18.71 & & - & - & - \\
\hline
\end{tabular}
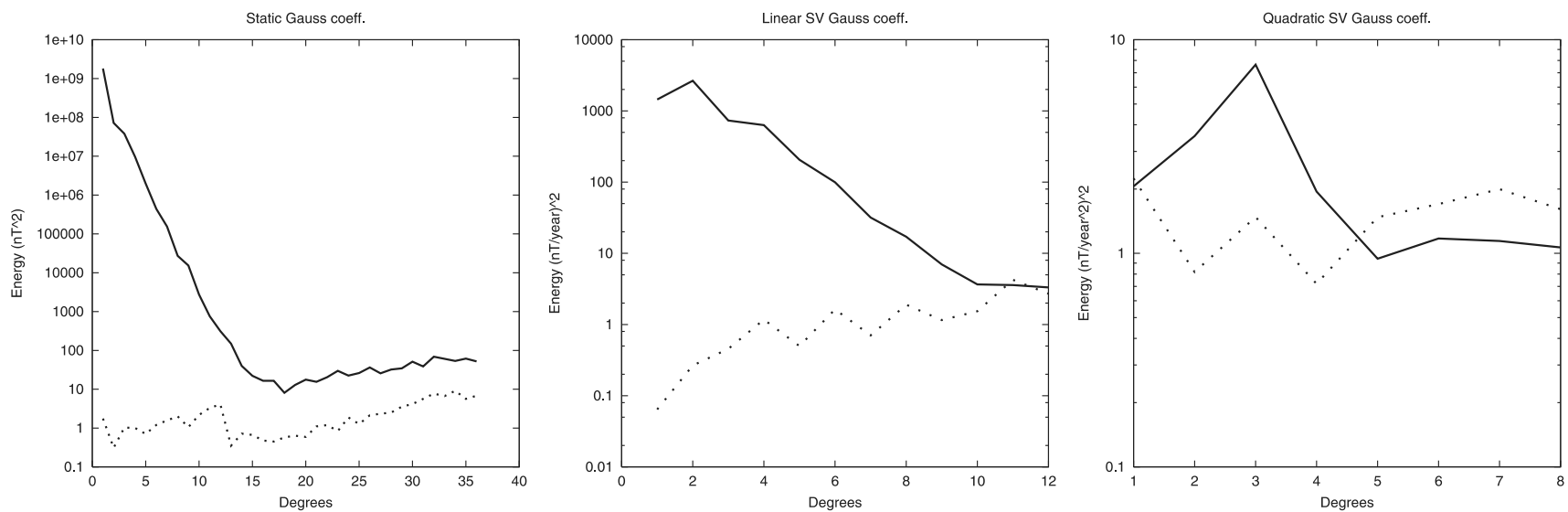

Fig. 1. Power spectrum plots of the parent model (solid line) and its difference with respect to the equivalent model built without observatory data (dotted line). From left to right: constant coefficients, their linear variation with time and their acceleration.
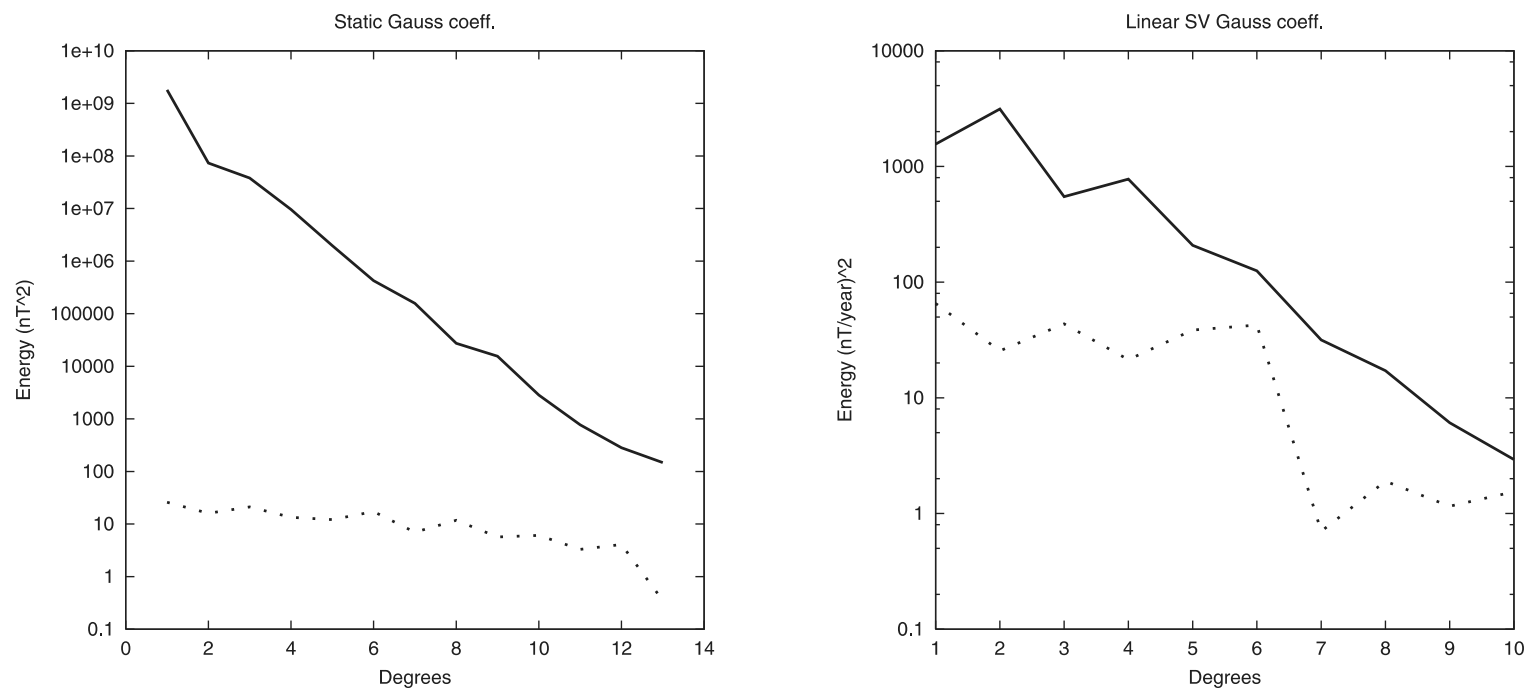

Fig. 2. Power spectrum plots of the parent model (solid line) and its difference with respect to the equivalent model built without observatory data (dotted line). Left: main field model for 2005.0. Right: Secular variation model for 2007.5.

scheme, their inverses were put in diagonal weight matrices that left and right multiplied the covariance matrix.

Let $\boldsymbol{C}_{d}$ be the data covariance matrix multiplied by the weight matrices above. This matrix is real and symmetric and can therefore be reduced to a real diagonal matrix by orthogonal rotation:

$$
\boldsymbol{C}_{d}=\boldsymbol{A}^{t}\left(\boldsymbol{S}^{t} \boldsymbol{S}\right)^{-1} \boldsymbol{A}
$$

where the superscript ${ }^{t}$ denotes the transpose and the superscript $^{-1}$ the inverse, $S$ is a real diagonal matrix whose elements are the inverse of the weighted SDs and $\boldsymbol{A}$ is the 
Boulder: 39.943Deg N, 254.767Deg E
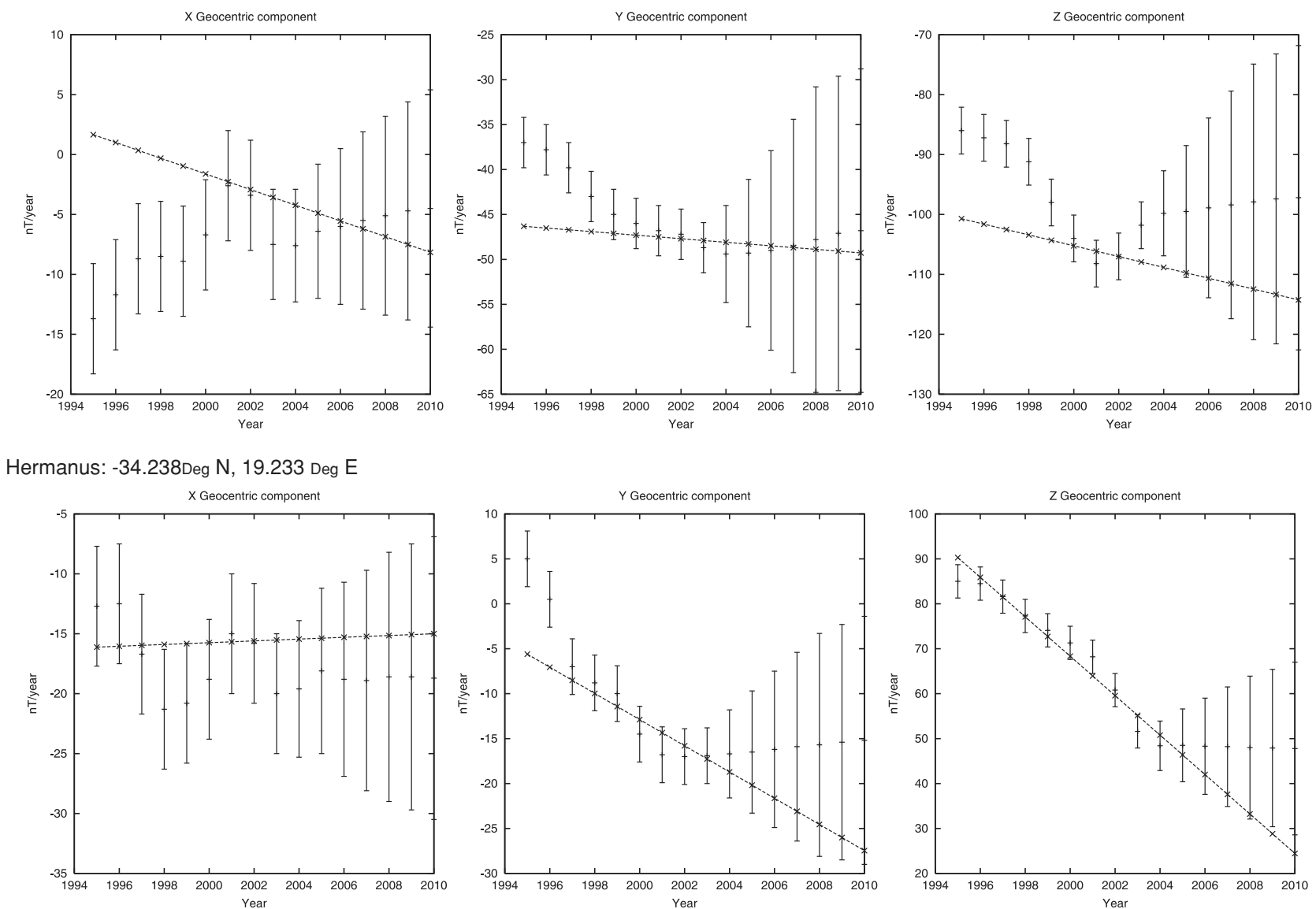

Memambetsu: 43.725Deg N, 144.183Deg $E$
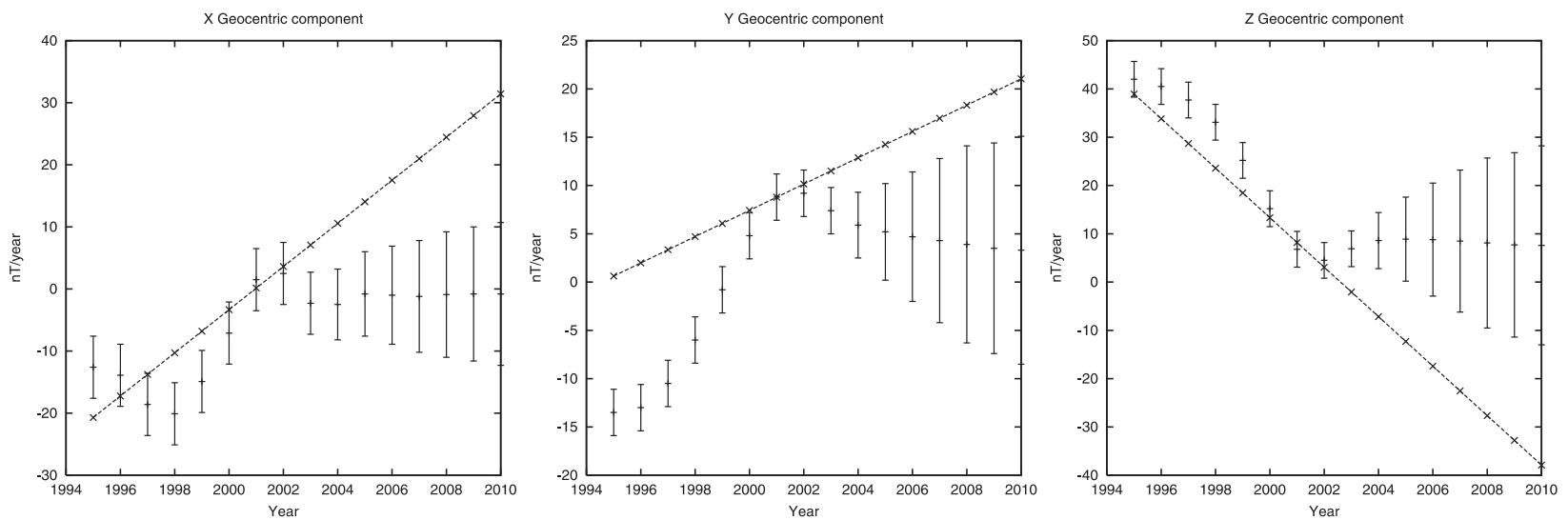

Fig. 3. Comparison of recorded or predicted secular variation annual means (points with error bars) and secular variation estimated from the parent model (solid lines) extrapolated outside (1999.0-2004.58). Values for Boulder, Hermanus and Memambetsu observatories are shown.

inverse of the rotation matrix. (The notation used is consistent with that of Olsen, 2002).

\section{Model Estimation}

We estimated the model parameters in four iterations, fitting the data using the classic least squares approach i.e. assuming a Gaussian distribution of residuals (Lesur et al., 2005).

Two models were derived; one (the parent model from which the BGS candidate is derived later on) used the full data set, and the second used only the satellite data set. The number of data, mean and the rms misfits to the data for the resulting models are given in Table 1 . The $X, Y$ and $Z$ components are oriented North, East and down respectively and $N$ is the number of data values. The " $F$ observatory" data are the observatory projected vector values.

There is not an excessive increase of satellite data rms when observatory data are introduced but the mean value of the satellite scalar data residuals is significantly increased. Therefore, the effect of introducing observatory data is most likely to be seen at high latitude. Figure 1 shows the power spectra of the constant part, the linear secular variation and the acceleration in time of the parent model Gauss coefficients for time 2002.0. Also shown are the power spec- 
tra of their differences with the Gauss coefficients obtained without observatory data. The observatory data directly contribute to the estimation of the secular variation model, therefore, the differences in $s v$ power spectrum between the two models are important. These differences are particularly large for degree 11 and 12 where the validity of $s v$ Gauss coefficients is probably questionable. These same observatory data only affect the constant Gauss coefficients via the constraint imposed on the $s v$ model and therefore they do not have a large effect on these coefficients: the power spectrum of the difference between the models stays small. There is a step after degree 12 because linear secular variation is not modelled above this degree. For the acceleration the degrees 2, 3 and 4 are obviously the most reliable. However the acceleration is controlled mostly by the data at the beginning and end of the time span. There are few satellite data in 1999 and no observatory data after 2004. Therefore any comparison of the acceleration in these two models may not be reliable.

\section{Extrapolation to 2005.0 and 2007.5}

The model resulting from the above process, when data from all satellites and observatories are used, is valid only from 1999.0 to 2004.58 and therefore has to be extrapolated forward in time. The coefficients for the main field model at 2005.0 and secular variation model at 2007.5 were calculated using Eq. (2). For this extrapolation, the $\dot{g}_{l}^{m}$ and $\dot{g}_{l}^{m}$ coefficients for degree 11 and above, as well as the $\ddot{g}_{l}^{m}$ and $\ddot{h}_{l}^{m}$ for degree 7 and above, were set to zero. Figure 2 shows the power spectra of these two models and the power spectra of their differences with respect to the models built using the same process but without hourly mean observatory data. For the 2005.0 main field model, the Gauss coefficient differences have a power spectrum that is relatively constant with increasing degree, but one order of magnitude larger than for 2002.0. This increase between 2002.0 and 2005.0 is the cumulative effect of the differences in the secular variation and acceleration models. For the 2007.5 secular variation model, the Gauss coefficient differences have a power spectrum that drops at degree 7 due to the truncation of the acceleration model. By applying this truncation we avoid having secular variation models too different in 2007.5 even if we recognise, as above, that the acceleration model built without the hourly mean observatory data is not very robust. The 2005.0 main field model was then truncated to degree 13 to produce the main field candidate for the IGRF-10.

Figure 3 shows secular variation annual means at three observatories, measured up to 2003.0, and thereafter, predicted using linear filters (Macmillan and Quinn, 2000). Also shown are the estimated secular variation values computed from the parent model. The measured annual means and the spot values estimated for the reference date of the model (2002.0) agree. However, there is very little agreement seen when the parent model is extrapolated backward in time, giving some indication of how inaccurate the forward extrapolation of the parent model might be. The predicted annual means are likely to be more accurate than the secular variation values estimated from the parent model simply because of the long time span covered by the observatory data. Therefore these annual means have to be incor-

\section{North component}

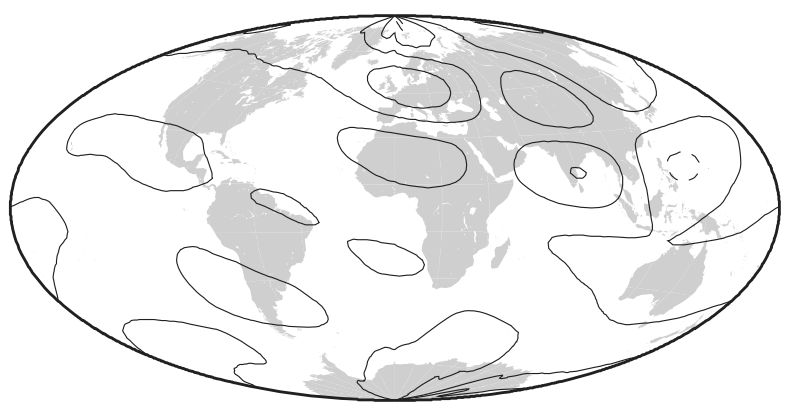

\section{East component}

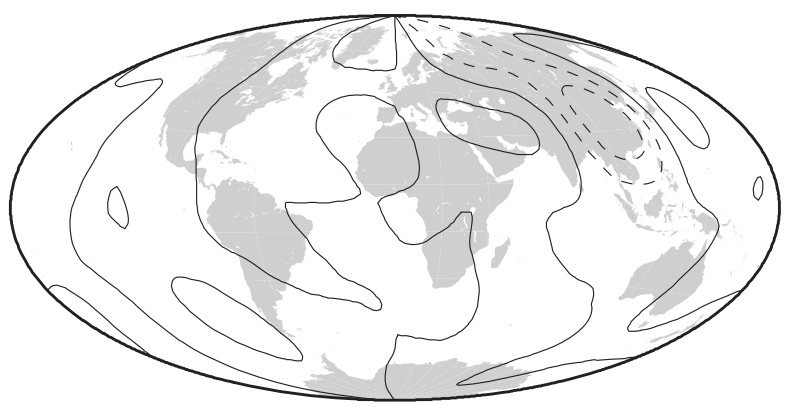

Vertical component

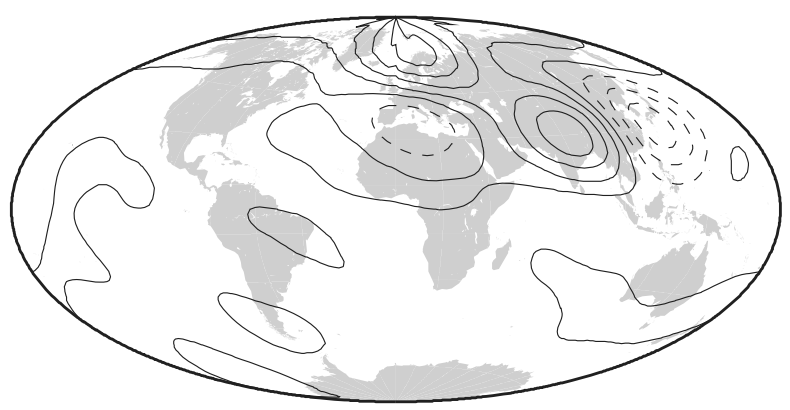

Fig. 4. Contoured differences between the parent secular variation model in 2007.5 and the BGS secular variation IGRF-10 candidate showing the effect of introducing annual mean data. Contour interval $1 \mathrm{nT} /$ year, negative solid, positive dashed.

porated in the estimation of the candidate secular variation model. To do this, the 2007.5 secular variation model was used to produce a synthetic data set on a $6371.2 \mathrm{~km}$ radius sphere, composed of vector data located at the centres of equal-area cells whose size at the equator was $5^{\circ}$ in latitude and longitude. This data set, comprising 1654 vector values, was then augmented by the annual mean secular variation predictions from 188 observatories. Each datum was weighted by the inverse of its estimated uncertainty. These estimated uncertainties are outputs of the linear prediction filter (Macmillan and Quinn, 2000) for the observatory annual mean secular variations. Examples of these estimates are shown in Fig. 3. For the synthetic data set, the estimated uncertainties are easily calculated from the formal standard deviations of the underlying model Gauss coefficients. However, these synthetic data were down-weighted 
because these formal standard deviations are known to be too small (Lowes and Olsen, 2004). Overall they were on average given higher weights than the annual mean observatory secular variation values for the candidate secular variation model not to be too different from the parent model. A spherical harmonic model of the secular variation with maximum degree 10 was then fitted to these data and truncated to degree 8 to produce the BGS secular variation candidate model for the IGRF-10. Figure 4 shows the contoured differences between the parent 2007.5 secular variation model truncated to degree 8 and the IGRF-10 BGS secular variation candidate for 2005.0 to 2010.0. The largest differences are less than $5 \mathrm{nT} /$ year and are observed in the vertical component, mainly over East Asia.

\section{Conclusion}

We have produced a geomagnetic field model for year 2005.0 and an associated secular variation model for the period 2005.0 to 2010.0. These models are not only based on satellite data but also on observatory hourly mean values and linearly predicted annual mean secular variation. We believe the model obtained provides a reliable description of the main magnetic field.

Acknowledgments. The staff of magnetic observatories around the world, the Ørsted and Champ data centres and the World Data Centres in Copenhagen, Kyoto and Edinburgh are thanked for providing the data on which this study depends. The IMF data are from the Advance Composition Explorer (ACE) spacecraft and made available through OMNIWeb (courtesy NASA). This paper is published with the permission of the Executive Director of the British Geological Survey (NERC).

\section{References}

Hapgood, M. A., Space physics coordinate transformation: A user guide, Planetary and Space Science, 40, 711-717, 1992.

Holme, R., Modelling of attitude error in vector magnetic data: application to Ørsted data, Earth Planets Space, 52, 1187-1197, 2000.

Langel, R. A. and R. H. Estes, Large-scale, near-Earth magnetic fields from external sources and the corresponding induced internal field, $J$. Geophys. Res., 90, 2487-2494, 1985.

Lesur, V., S. Macmillan, and A. Thomson, Magnetic field model with daily variations of the magnetospheric field and its induced counterpart in 2001, Geophys. J. Int., 160, 79-88, 2005.

Lowes, F. J. and N. Olsen, A more realistic estimate of the variances and systematic errors in spherical harmonic geomagnetic field models, Geophys. J. Int., 157, 1027-1044, 2004.

Macmillan, S. and J. M. Quinn, The 2000 revision of the joint UK/US geomagnetic field models and an IGRF2000 candidate model, Earth Planets Space, 52, 1149-1162, 2000.

Maus, S. and P. Weidelt, Separating magnetospheric disturbance magnetic field into external and transient internal contributions using 1D conductivity model of the Earth, GRL, 31(12), L12614, 10.1029/2004GL020232, 2004.

McLean, S., S. Macmillan, S. Maus, and V. Lesur, A. Thomson, and D. Dater, The US/UK World Magnetic Model for 20052010. NOAA Technical Report NESDIS/NGDC-1 available at www.geomag.bgs.ac.uk/documents/wmm_2005.pdf and www.ngdc. noaa.gov/seg/WMM/data/TRWMM_2005.pdf, 2004.

Olsen, N., A model of the geomagnetic field and its secular variation for epoch 2000 estimated from Ørsted data, Geophys. J. Int., 149, 454-462, 2002.

Sabaka, T. J., N. Olsen, and R. A. Langel, A comprehensive model of the quiet-time, near-Earth magnetic field: phase 3, Geophys. J. Int., 151, 32-68, 2002.

V. Lesur (e-mail: V.Lesur@bgs.ac.uk), S. Macmillan (e-mail: S.Macmillan@bgs.ac.uk), and A. Thomson (e-mail: A.Thomson@ bgs.ac.uk) 